\title{
A Modified Methodology for Retrieval of Arbitrarily Video Objects
}

\author{
Mohammed J. Mohammed \\ College of Engineering, Nawroz University, Duhok, Kurdistan Region - Iraq
}

\begin{abstract}
Abstract- The increasing availability of object-based video content requires new technologies for automatically extracting and matching of the low level features of arbitrarily shaped video. In this paper, methods for shape retrieval of arbitrarily shaped video objects are proposed. Along with still shape features the shape deformations that may occur in an object's life span is also taken. In this paper a novel method is used for measuring shape similarities between arbitrarily shaped video objects by frames of the video objects.
\end{abstract}

KEYWORDS : object-based, video, extracting, shape similarities, video objects.

\section{INTRODUCTION}

The advancements in technologies for extracting video objects in a scene, such as cameras equipped with depth. sensors that capture three-dimensional (3-D) video and naturally segmented objects, combined with the Advancements in video object segmentation technologies, and efficient object-based video representations availability, such as MPEG-4 results in increased availability of arbitrarily shaped digital video content. The most recent MPEG- video coding standard, MPEG-4 offers an object based representation of video, where individual video objects (Vos) are coded into separate bit streams. In the MPEG-4 terminology, temporal instances of video objects are referred to as video object planes (VOPs). Similar to key frames, key VOPs can be used for visual summarization of the video object content in an object-based framework,. Shape matching is an important part of content-based visual data retrieval as how humans recognize objects primarily by their shape. Much effort has been taken for finding the shape features and similarity matching techniques that closely resemble human perception. In the existing video object retrieval systems, shapes of objects are represented with one set of features,

Academic Journal of Nawroz University

(AJNU) Volume 7, No 3 (2018).

Received 16 April 2018.

Regular research paper : Published 20 June 2018

Corresponding author's e-mail : Mjm562000@yahoo.com

Copyright (C2018 Mohammed J. Mohammed.

This is an open access article distributed under the Creative Commons Attribution License. generally Obtained by averaging the features that represent each temporal instant of video objects. While such a Representation would work well for video objects that have constant shapes during their life spans, it is insufficient in the cases where the shapes of a video object changes. In this paper, the above described problem is overcome by a novel method for measuring shape similarities between two arbitrarily shaped video objects by comparing the Low-level still shape features of the representative frames of the video objects. A video object sequence consists of video objects; A video object sequence consists of video object planes (VOPs) that describe the shape and texture that the object has in a particular instant. As the shape of a video object could vary in the temporal dimension, the shape deformation's type that an object goes through could offer valuable information about the object's occlusion, local motion, articulated parts, and elasticity. In these paper domain-independent descriptors for representing the local motion of arbitrarily shaped video objects is taken. These descriptors were based on the fact that any significant motion of video objects is very likely to result in changes in the object's shape and the variance of this change can potentially describe the type of object motion. As digital video is mostly available in compressed forms, most object-based video retrieval systems perform analysis in the uncompressed domain. In this paper also the compressed domain retrieval of MPEG-4video objects has been addressed. The MPEG-4 compressed domain framework is employed mainly because which helps to separate the segmentation problem from the retrieval problem. This method offers good retrieval Three main sections in this paper are presented as follows : - 
\#. form matching of video objects is completed by a similarity measure that's supported matching a set of representative video object planes of a video object.

\#. Then extraction And matching of the new descriptors representing video object motion is given. The shape of each temporal instant of a video object is diagrammatical With Fourier descriptor These coefficients conjointly amendment as the form of video object changes with time Then another descriptor Angular Circular native Motion Descriptor is computed to capture the placement in addition because the type of object motion.

\#. During this final section descriptors are computed in the MPEG-4 compressed domain. A rough approximation of the video object contour by shaping intra coded form B locks because the contour purpose is obtained.

\section{MATERIALS AND METHODOLOGY}

The first step in retrieving the willy-nilly formed video objects is assortment of information. The information contains fifty MPEG-4 video object streams, together with quite twenty arbitrarily formed video objects. Once aggregation the Database the video objects square matched supported its form initially for matching the video objects by its form a Distance live has been utilized. The geometer Distance is measured as : -

$$
\begin{gathered}
\mathrm{d}\left(\mathrm{VO}_{\mathrm{A}}, \mathrm{VO}_{\mathrm{B}}\right)= \\
1 / N \sum_{k}^{N} \min _{V O P_{b} \in O_{B}}\left\{d_{v o p}\left(V^{\prime} P_{a k}, V O P_{b}\right)\right\}
\end{gathered}
$$

$\mathrm{N}$-number of representative VOPs of VOA, VOPak, is the kth representative video object plane of VOA, within the on top of distance live for each VOP of VOA, the tiniest distance to any representative VOP in VOB is found. After then the add of those distances is split by the quantity of VOPs in VOA to search out the space between 2 video objects. The geometer distance between 2 VOPs is calculated as : -

$$
d_{\text {vop }}\left(V O P_{a}, V O P_{b}\right)=\left\|\underset{R_{a}}{\longrightarrow}-\underset{R_{b}}{\longrightarrow}\right\|
$$

Where, $\underset{R_{a}}{\longrightarrow}$ and $\underset{R_{b}}{\longrightarrow}$ are the feature vectors of the

$\operatorname{VOP}_{\mathrm{a}}$ and $\mathrm{VOP}_{\mathrm{b}}$ respectively.

The on top of computed distance live is, asymmetric. so the ultimate distance is calculated as DVO (VOA, $\mathrm{VOB})=\max$,

\section{$\left\{\mathrm{d}_{\mathrm{ov}}\left(\mathrm{VO}_{\mathrm{A}}, \mathrm{VOB}\right), \mathrm{dvo}\left(\mathrm{VO}_{\mathrm{B}}, \mathrm{VO}_{\mathrm{A}}\right)\right\}$}

As the forms of the video objects vary with time, their native motion is delineate victimization 2 main descriptors : -

1. Fourier constant primarily based native motion Descriptor.

2. Angular Circular native Motion Descriptor. local motion supported the variances of the Fourier Fourier constant primarily based native motion Descriptor finds the Coefficients. The variance vector is computed as follows : -

$$
\underset{F \sigma^{2}}{\longrightarrow}=1 / K \sum_{k=0}^{K}\left(\underset{F_{k}}{\longrightarrow}-\frac{}{F_{\mu}}\right)^{2}
$$

and the mean Fourier vector is computed as, $\underset{F_{\mu}}{\longrightarrow}=1 / K \sum_{k=0}^{K} \underset{F_{k}}{\longrightarrow}$

Where $\mathrm{K}$ is the number of VOPs in a video object. $\underset{F_{k}}{\longrightarrow}$ is the kth VOPs Fourier vector. $\underset{F_{\mu}}{\longrightarrow}$ is the average of the feature vectors of all VOPs in a video object.

Every element of $\underset{F_{\sigma} 2}{\longrightarrow}$ is divided by $\max _{V O P_{k} \in V O}\left\{F_{1}\left(V O P_{k}\right)^{2}\right\}$, where $\mathrm{F}_{1}\left(\mathrm{VOP}_{\mathrm{k}}\right)$ is the first nonzero Fourier constant of the kth VOP.The magnitudes of. the complicated coefficients are used as motion options. Angular Circular native Motion Descriptor finds the situation as well because the form of object motion. Info regarding the shape deformation gift in a very video object is obtainable by the variance of the object area. Based on this the shape mask of the video object is divide in to $\mathrm{M}$ angular and $\mathrm{N}$ circular segments and the variances of the pixels that fall into each segment describes the local motion. The variance is computed as follows : -

$$
\sigma_{n, m}^{2}=1 / S(n, m)^{2} K \sum_{k=0}^{K-1}\left(P_{n, m}-\mu_{n, m}\right)^{2}
$$

where $\mathrm{K}$ is the number of the VOPs of the Video object, $\mathrm{VOP}_{\mathrm{k}}$ is the binary shape mask of the video object at that instant.

$$
\mu_{n, m}=1 / K \sum_{k=0}^{K-1} P_{n, m}
$$

$P n, m=\sum_{\theta=\theta m \rho=\rho n}^{\theta m+1} \sum_{\rho n+1}^{\rho n} V O P_{k}(\rho, \theta)$

$\operatorname{VOP}_{\mathrm{k}}(\rho, \theta)$ is the binary shape mask value in VOPk at position $(\theta, \rho)$ at the center of $\operatorname{VOP}_{\mathrm{k}} . \mathrm{S}(\mathrm{n}, \mathrm{m})$ is the area, $\theta_{\mathrm{m}}$ is the start angle and $\rho_{\mathrm{n}}$ is the start radius of the angular circular segment $(\mathrm{n}, \mathrm{m})$. They are defined by, $\mathrm{S}$ (n, $\mathrm{m})=\Pi\left(\rho_{\mathrm{n}+1}^{2}-\rho_{\mathrm{n}}^{2}\right) / \mathrm{M}$, where $\theta_{\mathrm{m}}=\mathrm{m} * 2 \Pi / \mathrm{M}$, and $\rho_{\mathrm{n}}=\mathrm{n}^{*} \rho_{\max } / \mathrm{N}, \rho_{\max }=\max _{V O P_{k} \in V O}\left\{\rho \mathrm{VOP}_{\mathrm{k}}\right\}$ where $\mathrm{M}$ and $\mathrm{N}$ where $\mathrm{K}$ is the number of the VOPs of the Video object $F_{k}, V O P_{K}$

Is the kth video -- are the angular and circular sections, $V O P_{K}$

object plane of the video object, and $\rho V O P_{K}$ is the 
radius of the circle around $V O P_{K}$

matrix $\mathrm{R}$ is formed by the variance $\sigma_{m, n}^{2}$,

Then the local motion feature

Then the descriptors in the MPEG-4 compressed domain are computed by shape coding method. The video object contour is obtained by defining the intracoded shape blocks as the contour points.

\section{HOW IT WORKS}

In this paper the system for retrieving the shape of video objects works as follows and it consists of the following step : -

1. Shape Matching of video objects.

2. Local Motion Descriptors Based on Shape Deformations.

3. Computation of the Descriptors in the MPEG-4 compressed domain.

The first step, shape matching of video objects is done by a similarity measure between the video objects. Kmeans clustering is performed before computing the similarity distance.

In the second step, two main descriptors are computed.

a. Fourier Coefficient Based Local motion Descriptor finds the local motion based on the variances of the Fourier Coefficients. The variance vector and the mean Fourier vector is computed.

b. Angular Circular Local Motion Descriptor finds the location as well as the type of object motion. The variance and mean vector is computed here. Then in the final step, shape-coding method is used to compute the descriptors. This is done by first dividing the video object into $16^{*} 16$ blocks then the blocks are transmitted that are inside the VOP as opaque, the blocks that are outside the VOP as transparent, and the boundary blocks, i.e., blocks that contain pixels both inside and outside the VOP, as intracoded with context-based arithmetic coding. Then a rough approximation of the video object contour by defining the intracoded shape blocks as the contour points is obtained. Defining the intra and opaque coded blocks as inside the shape approximates the object's binary shape mask. Here, the object's area is approximated finally using the formula as, $\mathrm{A}=\mathrm{O}+0.5 \mathrm{I}$ where $\mathrm{O}$ is the number of opaque coded shape blocks and I is the number of intracoded shape blocks.

\section{COMPARISON WITH EXISTING SYSTEMS}

In the existing video object retrieval systems, shapes of objects are represented with one set of features, generally obtained by averaging the features that represent each temporal instant of video objects.

While such a representation would work well for video objects that have constant shapes during their lifespan, it is insufficient in the cases where the shape of a video objects changes. In this paper, this problem is overcome by using a novel method for measuring shape similarities between two arbitrarily shaped video objects by comparing the low-level still shape features of the representative frames of the video objects. As the shape of a video object could vary in the temporal dimension, the type of shape deformations that an object goes through could offer valuable information about the object's occlusion, local motion, articulated parts, and elasticity. In a typical retrieval system, a feature vector is formed for each object and then the similarities between objects are found by computing the distance between these feature vectors. A video object sequence consists of video object planes (VOPs) that describe the shape and texture that the object has in a particular instant. In the existing systems, the feature vector of one key object plane is used to represent the whole video object sequence or by computing the average of the feature vectors belonging to all object planes. Such techniques work well for representing the color content of video objects, which tend to remain somewhat consistent during an object's lifespan. However, these techniques can fail to accurately represent an object's shape content, considering that the object's shape may change significantly during its existence. In this paper the above problem is overcome by using a similarity measure that is based on matching a subset of representative video object planes of a video object.

\section{CONCLUSION}

Matching of video objects based on their shape information is a very important component of any video object retrieval system. The possible variations in a video object's shape in time makes matching of objects using theirs shapes a

challenging task Thus, in this paper shape matching via comparing a subset of representative planes of video objects is performed which outperformed the commonly used averaging technique. In this paper, the motion of objects based on variations of still shape descriptors is also described. The descriptors have been shown to successfully classify local object motion. The speed of the motion of these descriptors is not captured. Here shape and local motion descriptors are computed using the low-resolution shape data obtained from the MPEG-4 bit stream.

\section{REFERENCES}

1. H.T.Nguyen, M. Worring, and A.Dev, "Detection of moving objects in video using a robust motion similarity measure," IEEE Trans. Image Process., vol. 9, no.1, pp. 88-101,Jan. 2000.

2. MPEG-4 Video Group," Coding of Audio-Visual Objects : Video SO/IEC 14 496-2, 2000.

3. S. F. Chang, W. Chen, H. J. Meng, H. Sundaram, and D. Zhong, "A Fully automated content-based video search engine supporting spatiotemporal queries," IEEE 
Trans. Circuits Syst. Video Techno vol. 8, no. 5, pp.602615, Sep. 1998.

4. Y. Deng and B. S.Manjunath, "NeTra-V : Toward an object-based video representation," IEEE Trans. Circuits Syst. Video Technol., vol8 pp.616-627, Sep. 1998.
5. B. Erol and F.Kossentini, "Automatic key video object plane selection using the shape information in the MPEG-4 compressed domain IEEE Trans. Multimedia, vol. 2, no. 2, pp. 129-138, Jun. 2000.

\section{FLOWCHART}

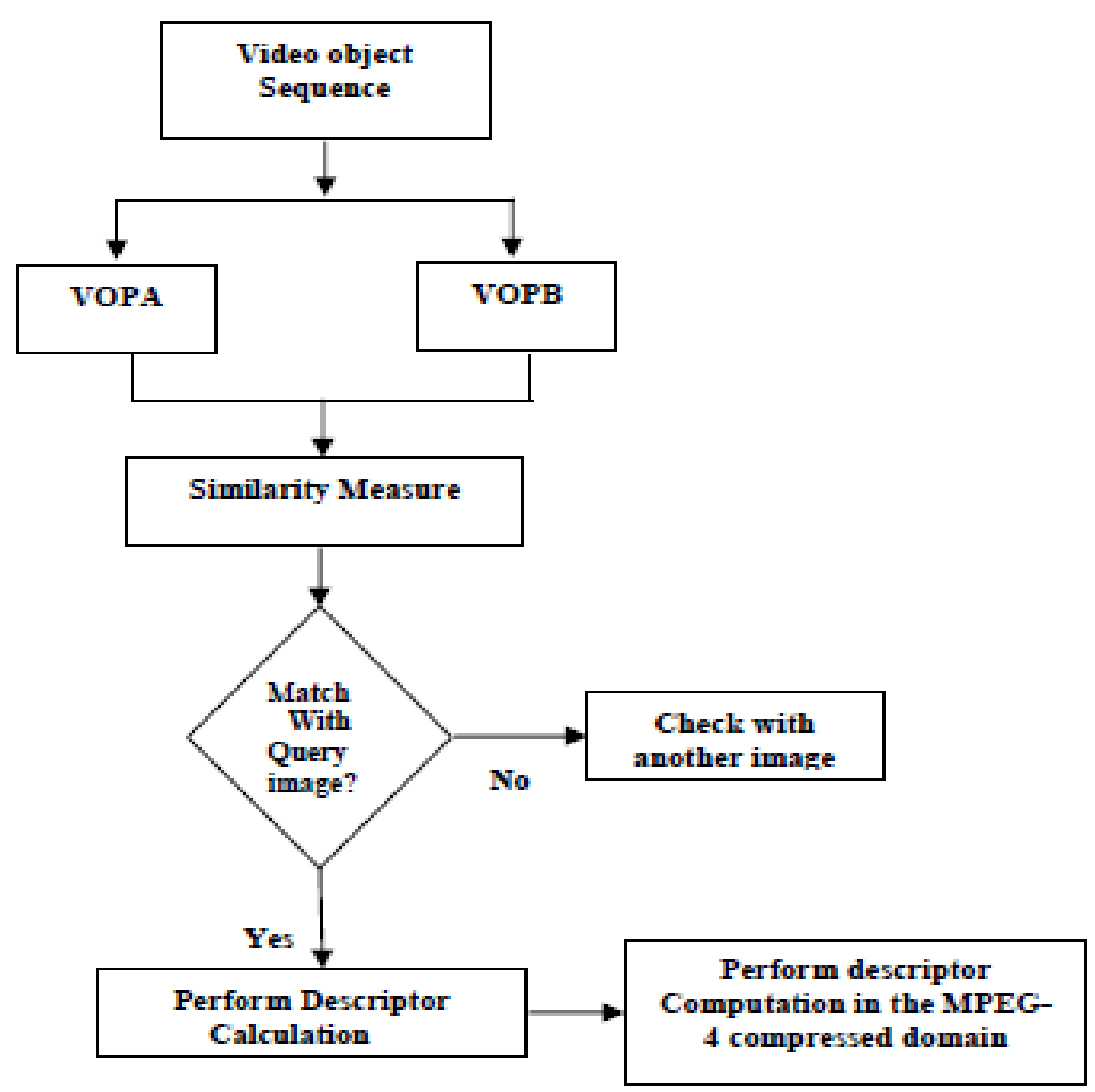

